\title{
DISPERSION RELATIONS, STORED ENERGY AND GROUP VELOCITY FOR ANISOTROPIC ELECTROMAGNETIC MEDIA*
}

\author{
BY \\ H. KURSS \\ Adelphi University
}

\begin{abstract}
The Hermitian and skew-Hermitian components of the susceptibility matrix of a general linear electromagnetic medium are represented as Hilbert transforms of each other. These so-called dispersion relations lead to a priori inequalities which must be satisfied by the susceptibility of a passive medium in a frequency interval in which the medium is lossless. One such inequality states that the stored energy density for a given $\mathbf{E}(\omega)$ and $\mathbf{H}(\omega)$ is always greater than in free space. This is also verified directly from the usual gyrotropic susceptibilities of ferrites and plasmas.

The group velocity for an eigenwave or mode of a structure with one, two or three independent translational symmetry vectors is shown to be, in general, an average Poynting vector divided by an average stored energy density. This formula is then combined with the above inequality for the stored energy density to show that the magnitude of the group velocity is less than $c$, the velocity of light in free space.
\end{abstract}

I. Introduction. A main point of the present paper is to extend some results, known for isotropic media [1], to the case of general anisotropic media. Moreover, some of the discussion, for example that relating to group velocity, is novel even when specialized to the isotropic case.

For simplicity as well as for generality the discussion is phrased for a medium where

$$
\left[\begin{array}{l}
D(\omega) \\
B(\omega)
\end{array}\right]=\gamma(\omega)\left[\begin{array}{l}
E(\omega) \\
H(\omega)
\end{array}\right]
$$

and none of the components of the matrix $\gamma(\omega)$ are necessarily zero. (The susceptibility of such a medium is defined to be $\gamma(\omega)-\gamma_{0}$ where $\gamma_{0}$ is the value of $\gamma(\omega)$ in free space.) Such a general medium, often called a Tellegen medium, is referred to in a number of recent works [2], [3], [4].

As in the isotropic case, a consequence of causality and time invariance is that the susceptibility, $\gamma(\omega)-\gamma_{0}$, satisfies an integral equation with (Cauchy) Kernel $1 /(i \pi(x-\omega)$ ), where the range of integration of $x$ is from $-\infty$ to $+\infty$. One can separate this integral equation into its real and imaginary components. The resulting equations [1], [5], called dispersion relations, state that the real and imaginary components of the susceptibility matrix, in their dependence upon frequency, are Hilbert transforms of each other. However, in the anisotropic case one has the additional possibility of separating the integral equation into its Hermitian and skew-Hermitian components. This leads to the new dispersion relations stated in Sec. III. These are particularly significant with regard

*Received January 9, 1967; revised manuscript received June 6, 1967. This research was completed under National Science Foundation Grant No. GP-2761. A preliminary version was presented at the International Conference on Microwaves, Circuit Theory and Information Theory, Tokyo, Japan, Sept. 7-11, 1964. 
to passivity considerations since the skew-Hermitian component of $\gamma$ is a measure of the loss in the medium.

It is physically important to consider the case of a medium which is lossless for frequencies in an interval $\Delta$. Such an interval is referred to as a transparency interval [1]. It is noted in Sec. IV that the dispersion relations do not restrict the location of $\Delta$. However, the behavior of $\gamma(\omega)$ in such a transparency interval is constrained by the inequalities indicated in Sec. IV. One such inequality is interpreted in Sec. V as stating that the stored energy density for a given $\mathbf{E}(\omega)$ and $\mathbf{H}(\omega)$ is greater than in free space. This inequality is then verified for the usual susceptibilities of ferrites [6] and plasmas [7].

Recent papers have proved the equivalence of group velocity and velocity of energy transport for the modes of special structures. For example, the literature contains discussions for plane waves in a uniform medium [8], [9], [10]; modes in a uniform waveguide [10], [11]; modes in a periodically loaded waveguide containing isotropic media [12]; Bloch waves in a crystalline medium [13]; surface waves in a plane stratified medium [14], etc. In Sec. VI of the present paper, the above discussions are unified and generalized so as to apply to the modes of a general structure with one, two or three independent translational symmetry vectors $a_{i}$. (The mode is characterized by a propagation vector $\mathbf{k}$ in the space, $\mathcal{L}$, spanned by the $\mathbf{a}_{i}$ by means of the phase-shift boundary conditions

$$
\boldsymbol{\Phi}\left(\mathbf{r}+\mathbf{a}_{\mathbf{i}}\right)=\exp \left(i \mathbf{k} \cdot \mathbf{a}_{i}\right) \boldsymbol{\Phi}(\mathbf{r})
$$

imposed upon both the $\mathbf{E}$ and $\mathbf{H}$ field vectors.) A key item for this purpose is an identity of which various versions exist in the literature [10], [11], [14], [15]. A proof for the case of a general Tellegen medium is contained in the Appendix.

Another contribution of Sec. VI is the proof that the group velocity has a magnitude which, in general, is less than $c$, the velocity of light in free space. (This result was previously proved only for the case of plane wave modes in an isotropic medium [1].) The proof is based upon the above inequality for the stored energy density combined with a simple upper estimate for the magnitude of the Poynting vector.

II. The susceptibility matrix. We shall employ the Maxwell equations in MKS units, i.e.,

$$
\nabla \times \mathcal{E}(t)=-\partial \boldsymbol{B}(t) / \partial t, \quad \nabla \times \mathfrak{H C}(t)=\partial \mathfrak{D}(t) / \partial t
$$

where $\boldsymbol{B}(t)$ and $\boldsymbol{D}(t)$ include the effects of any magnetic and electric currents which may be present. The constraint imposed by the medium will be taken to be

$$
\begin{aligned}
& \boldsymbol{D}(t)=\hat{\boldsymbol{\epsilon}} \mathcal{E}(t)+\hat{\xi} \boldsymbol{\mathcal { C }}(t), \\
& \boldsymbol{B}(t)=\hat{\eta} \mathcal{E}(t)+\hat{\mu} \mathcal{H}(t),
\end{aligned}
$$

where, for a linear medium, $\hat{\epsilon}, \hat{\mu}, \hat{\xi}$ and $\hat{\eta}$ are linear operators.

It is convenient to replace (2) by the single equation

$$
\psi(t)=\hat{\gamma} \phi(t)
$$

where

$$
\Psi(t)=\left[\begin{array}{l}
\mathfrak{D}(t) \\
\boldsymbol{B}(t)
\end{array}\right], \quad \boldsymbol{\phi}(t)=\left[\begin{array}{r}
\mathcal{E}(t) \\
\mathfrak{F C}(t)
\end{array}\right]
$$


and

$$
\hat{\gamma}=\left[\begin{array}{cc}
\hat{\xi} & \hat{\xi} \\
\hat{\eta} & \hat{\mu}
\end{array}\right] .
$$

In practice the most common case is

$$
\hat{\xi}=0 \text { and } \hat{\eta}=0 .
$$

However, for simplicity as well as for generality the subsequent discussion is phrased in terms of the matrix $\hat{\gamma}$ without the restriction in (4). (One often refers to a medium in which $\hat{\xi}$ and $\hat{\eta}$ are not both zero as being a Tellegen medium. Thus (4) is said to characterize a non-Tellegen medium.)

In free space $\hat{\gamma}$ is simply the constant matrix

$$
\gamma_{0}=\left[\begin{array}{cc}
\epsilon_{0} I & 0 \\
0 & \mu_{0} I
\end{array}\right]
$$

where $I$ is the three-dimensional identity matrix and $\epsilon_{0}, \mu_{0}$ are real scalar constants. For later purposes we note here that in MKS units

$$
1 /\left(\mu_{0} \epsilon_{0}\right)^{1 / 2}=c
$$

where $c$ is the velocity of light in free space.

For a general linear, time-invariant, causal medium

$$
\hat{\gamma} \phi(t)=\gamma_{0} \phi(t)+\int_{0}^{\infty} g(\tau) \phi(t-\tau) d \tau,
$$

where $g(\tau)$ is a matrix which accounts for the memory retained by the medium of events which took place $\tau$ seconds in the past. Causality is incorporated into (7) by the fact that only positive values of $\tau$ occur in the integration, i.e., only the past affects the present. Since the fields $\psi(t)$ in (3) are real, $g(\tau)$ in (7) must be real.

Consider now the specialization of (7) and (3a) to the case of a time variation of angular frequency $\omega$. More precisely, let

$$
\boldsymbol{\psi}(t)=[\mathbf{\Psi}(\omega) \exp (-i \omega t)]_{r}, \quad \boldsymbol{\phi}(t)=[\boldsymbol{\Phi}(\omega) \exp (-i \omega t)]_{r}
$$

where $A$, denotes the real part of $A$. When (8) and (7) are substituted into (3) one obtains

$$
\mathbf{\Psi}(\omega)=\gamma(\omega) \mathbf{\Phi}(\omega)
$$

where

$$
\gamma(\omega)=\gamma_{0}+\int_{0}^{\infty} g(\tau) e^{i \omega \tau} d \tau .
$$

The matrix $\gamma(\omega)-\gamma_{0}$ is called the susceptibility of the medium.

A less compact but perhaps more familiar form of (9) results when one sets

$$
\boldsymbol{\Psi}(\omega)=\left[\begin{array}{l}
\mathrm{D}(\omega) \\
\mathbf{B}(\omega)
\end{array}\right], \quad \boldsymbol{\Phi}(\omega)=\left[\begin{array}{c}
\mathbf{E}(\omega) \\
\mathbf{H}(\omega)
\end{array}\right]
$$


and

$$
\gamma(\omega)=\left[\begin{array}{ll}
\epsilon(\omega) & \xi(\omega) \\
\eta(\omega) & \mu(\omega)
\end{array}\right]
$$

in (9).

From the fact that $g(r)$ is real one concludes from (10) that for real $\omega$

$$
\bar{\gamma}(\omega)=\gamma(-\omega)
$$

where $\bar{\gamma}$ denotes the complex conjugate of $\gamma$. In terms of the real and imaginary components of $\gamma, \gamma_{r}$ and $\gamma_{i}$, one can rewrite (12) as

$$
\gamma_{r}(\omega)=\gamma_{r}(-\omega), \quad \gamma_{i}(\omega)=-\gamma_{i}(-\omega) .
$$

Thus $\gamma_{r}$ is an even function of $\omega$ and $\gamma_{i}$ is an odd function of $\omega$. However, (12) and (13) do not simplify the subsequent discussion and are not employed further in this paper.

III. Dispersion relations. From (10) one concludes that for real $\omega, \gamma(\omega)$ satisfies the integral equation

$$
\gamma(\omega)-\gamma_{0}=\frac{1}{i \pi} f \frac{\gamma(x)-\gamma_{0}}{x-\omega} d x
$$

where the range of integration in (14), and in all subsequent such equations, is from $-\infty$ to $+\infty$ and where the stroke through the integral sign indicates that the integration over the singularity at $x=\omega$ is defined to be the Cauchy principal value. (One approach to deriving (14) from (10) is to apply the Cauchy integral theorem to $\left(\gamma(\omega)-\gamma_{0} /\left(\omega-\omega_{0}\right)\right.$ in the upper half plane [1]. Another approach is to exploit the fact, evident from (10), that $\gamma(\omega)-\gamma_{0}$ is the Fourier transform of a function of $\tau$ which vanishes for $\tau$ negative [16].)

When (14) is separated into its real and imaginary components one obtains the dispersion relations

$$
\begin{aligned}
\gamma_{r}(\omega)-\gamma_{0} & =\frac{1}{\pi} f \frac{\gamma_{i}(x) d x}{x-\omega}, \\
\gamma_{i}(\omega) & =-\frac{1}{\pi} f \frac{\gamma_{r}(x)-\gamma_{0}}{x-\omega} d x .
\end{aligned}
$$

An equally valid but more useful set of dispersion relations arises when one separates (14) into its Hermitian and skew-Hermitian components. More precisely,

$$
\gamma_{h}=\frac{\gamma+\gamma^{+}}{2}, \quad \gamma_{s}=\frac{\gamma-\gamma^{+}}{2 i}
$$

where $\gamma^{+}$, called the Hermitian adjoint of $\gamma$, is the complex conjugate of the transpose of $\gamma$. Then clearly

$$
\gamma=\gamma_{h}+i \gamma_{.}
$$

and (14) is equivalent to 


$$
\begin{aligned}
\gamma_{h}(\omega)-\gamma_{0} & =\frac{1}{\pi} f \frac{\gamma_{s}(x) d x}{x-\omega}, \\
\gamma_{s}(\omega) & =-\frac{1}{\pi} f \frac{\left(\gamma_{h}(x)-\gamma_{0}\right) d x}{x-\omega} .
\end{aligned}
$$

These dispersion relations state that the Hermitian and skew-Hermitian components of the susceptibility, $\gamma(\omega)-\gamma_{0}$, are Hilbert transforms of each other. (The usual trick [1] of employing (13) to convert the range of integration to $0<x<\infty$ instead of $-\infty<$ $x<\infty$ works for (15) but not for (18).)

A medium is said to be reciprocal if $\gamma$ is a symmetric matrix, i.e., if

$$
\gamma=\gamma^{T}
$$

where $\gamma^{T}$ denotes the transpose of $\gamma$. In this case, and only in this case, will the dispersion relations (15) and (18) be identical. When this is not the case, i.e., for a nonreciprocal medium, (18) is more meaningful than (15) with regard to passivity considerations since $\gamma_{s}(\omega)$ is a measure of the loss in the medium. This interpretation of $\gamma_{s}(\omega)$ is discussed below.

IV. Transparency intervals for passive media. The instantaneous power flow or Poynting vector, $\rho(t)$, is defined by

$$
\boldsymbol{P}(t)=\boldsymbol{\varepsilon}(t) \times \mathfrak{H C}(t) .
$$

For a simple harmonic variation of angular frequency $\omega$ the average value of $\rho(t)$, denoted by $\mathbf{P}$, is

$$
\mathrm{P}=\frac{\omega}{2 \pi} \int_{0}^{2 \pi / \omega} \boldsymbol{P}(t) d t
$$

For later purposes we note the well known result

$$
\mathbf{P}=\frac{1}{2}\left[(E(\omega) \times \overline{\mathbf{H}}(\omega)]_{r} .\right.
$$

This follows when one substitutes

$$
\boldsymbol{\varepsilon}(t)=(\mathbf{E}(\omega) \exp (-i \omega t))_{r} ; \quad \mathcal{H}(t)=(\mathbf{H}(\omega) \exp (-i \omega t))_{r}
$$

into (20) to obtain

$$
2 \boldsymbol{\rho}(t)=(\mathbf{E}(\omega) \times \mathbf{H}(\omega) \exp (-2 i \omega t))_{r}+(\mathbf{E}(\omega) \times \overline{\mathbf{H}}(\omega))_{r}
$$

and then notes that the double frequency term in (23) does not contribute to the average value of $\boldsymbol{P}(t)$ indicated in (21).

From (20) and the Maxwell equations (1),

$$
-\nabla \cdot \boldsymbol{P}(t)=\boldsymbol{E} \cdot \partial \mathfrak{D} / \partial t+\mathfrak{H} \cdot \partial \boldsymbol{B} / \partial t
$$

or in terms of $\phi(t)$ and $\psi(t)$ introduced in (3b)

$$
-\nabla \cdot p=\phi^{T} \cdot \partial \psi / \partial l
$$

and hence from (8)

$$
-\nabla \cdot \boldsymbol{\rho}=\frac{1}{2}\left[\left(\boldsymbol{\Phi}^{T} \cdot(-i \omega) \mathbf{\Psi} \exp (-2 i \omega t)\right)_{\boldsymbol{r}}+\left(\boldsymbol{\Phi}^{+} \cdot(-i \omega) \boldsymbol{\Psi}\right)_{\boldsymbol{r}}\right]
$$


In taking the average indicated in (21) the double frequency term in (24) does not contribute. One thus obtains from (24), with the aid of (9),

$$
-\nabla \cdot \mathbf{P}=\frac{1}{2}\left(\boldsymbol{\Phi}^{+} \cdot(-i \omega \gamma) \boldsymbol{\Phi}\right)_{r}=\frac{1}{2}\left(\Phi^{+} \cdot \omega \gamma \boldsymbol{\Phi}\right)_{i}=\frac{1}{2} \boldsymbol{\Phi}^{+} \cdot \omega \gamma_{s} \boldsymbol{\Phi}
$$

Since $-\nabla \cdot \mathbf{P}$ represents the average power dissipated in the medium, one concludes from (25) that a medium is passive at frequency $\omega$ if

$$
\boldsymbol{\Phi}^{+} \cdot \omega \gamma_{s} \boldsymbol{\Phi} \geq 0 \text { for arbitrary } \boldsymbol{\Phi} \text {. }
$$

A customary shorthand notation for (26) is

$$
\omega \gamma_{s}(\omega) \geq 0 \text {. }
$$

From (25) it is also evident that a medium is lossless at a frequency $\omega \neq 0$ if

$$
\gamma_{s}(\omega)=0 \text {. }
$$

A frequency interval in which $\gamma_{s}(\omega)=0$ is called a transparency interval. For definiteness let $\Delta$ be such an interval defined by, say, those $\omega$ for which $\left|\omega-\omega_{0}\right|<\delta$ where $\omega_{0}$ and $\delta$ are fixed real numbers. The dispersion relations do not impose any constraint on the location of $\Delta$. This can be seen as follows. Let $\Delta$ be arbitrarily given. Define $\gamma_{s}(\omega)$ to be zero in $\Delta$ and "arbitrary" outside of $\Delta$ to the extent that one can define $\gamma_{h}(\omega)-\gamma_{0}$ from the dispersion relation (18a). This specifies a $\gamma=\gamma_{h}+i \gamma_{s}$ consistent with dispersion relations (18) and with a transparency interval in $\Delta$. In particular, if $\Delta$ is selected to be the entire real frequency axis $-\infty<\omega<\infty$ one concludes from (18a) that

$$
\gamma(\omega)=\gamma_{0}
$$

In other words, free space is the only medium which is lossless at all frequencies.

A natural question which arises is the extent to which one can specify $\gamma_{s}(\omega)$ and $\boldsymbol{\gamma}_{\boldsymbol{h}}(\omega)$ simultaneously in a given frequency interval. In particular, for a medium which is passive at all frequencies to what extent is $\gamma(\omega)$ constrained by the dispersion relations for $\omega$ in a transparency interval $\Delta$ ? An obvious constraint from (18) is that $\gamma(\omega)$ must be an analytic function of $\omega$ for $\omega$ in $\Delta$. A further constraint is that $\gamma(\omega)$ must satisfy the inequality

$$
L\left(\omega\left(\gamma(\omega)-\gamma_{0}\right)\right) \geq 0 \text { for } \omega \text { in } \Delta
$$

where

$$
L=\sum_{i=1}^{N} a_{i}(\omega)(\partial / \partial \omega)^{i}
$$

and the $a_{i}(\omega)$ are any scalar functions of $\omega$ such that

$$
L(1 /(x-\omega)) \geq 0 \text { for } \omega \text { in } \Delta \text { and } x \text { not in } \Delta .
$$

In order to prove (30) one first notes that

$$
\omega /(x-\omega)=-1+x /(x-\omega)
$$

so that for $L$ defined by (31)

$$
L(\omega /(x-\omega))=L(x /(x-\omega))=x L(1 /(x-\omega)) .
$$


Hence, from (18a), for $\omega$ in $\Delta$,

$$
L\left(\omega\left(\gamma(\omega)-\gamma_{0}\right)\right)=\frac{1}{\pi} \int x \gamma_{.}(x) L\left(\frac{1}{x-\omega}\right) d x .
$$

The integration in (35) is effectively over all $x$ not in $\Delta$ since for $x$ in $\Delta, \gamma_{\bullet}(x)=0$ by hypothesis. But then in the integrand of (35) $L(1 /(x-\omega)) \geq 0$ by (32) and $x \gamma_{\bullet}(x) \geq 0$ by the passivity constraint. This then proves (30). Furthermore, if the strict inequality occurs in (32) then it is evident from (35) that the equality in (30) can only occur if the medium is free space. Thus, since

$$
(\partial / \partial \omega)^{2 N+1}(1 /(x-\omega))>0
$$

one concludes from (30) that for any medium different from free space

$$
(d / d \omega)^{2 N+1}\left(\omega\left(\gamma(\omega)-\gamma_{0}\right)\right)>0 \quad \text { for } \omega \text { in } \Delta .
$$

The case $N=0$ in (37) can be rewritten as

$$
(d / d \omega)(\omega \gamma(\omega))>\gamma_{0} \quad \text { for } \omega \text { in } \Delta .
$$

An interpretation of (38) in terms of stored energy density is given in the next section.

The case $N>0$ in (37) is

$$
(d / d \omega)^{2 N+1}(\omega \gamma(\omega))>0 \text { for } \omega \text { in } \Delta .
$$

This shows that if $\omega \gamma(\omega)$ is a polynomial in $\omega$ for $\omega$ in a transparency interval $\Delta$ then the medium must be free space. In particular, free space is the only medium which is nondispersive, i.e., for which $\gamma(\omega)$ is a constant, for $\omega$ in $\Delta$.

V. Stored energy. We will show that, as in the isotropic case, the average stored energy density in a medium at a frequency $\omega$ in a transparency interval is

$$
U=\frac{1}{4} \Phi^{+}(\omega) \cdot[(\partial / \partial \omega)(\omega \gamma(\omega))] \boldsymbol{\Phi}(\omega) .
$$

(Some authors, for example [14], employ RMS values for the field quantities and thus obtain a factor $1 / 2$ instead of $1 / 4$ in (40).)

One can derive (40) by a beat frequency approach [1], [18] or by a complex frequency (or damped harmonic frequency) approach [19]. We now sketch a variant of the latter approach for a general (Tellegen) medium. For this purpose let

$$
\boldsymbol{\phi}(t)=(\boldsymbol{\Phi}(p) \exp (-i p t))_{r}, \quad \psi(t)=(\mathbf{\Psi}(p) \exp (-i p t))_{r}
$$

where $p=\omega+i \sigma$ and $\sigma>0$.

At $t=-\infty$ there is no stored energy since the fields are zero there. Thus $u(t)$, the stored energy at time $t$, is obtained by an integration of the equation preceding (24) to be

$$
u(t)=\int_{-\infty}^{t} \phi^{T} \cdot \frac{\partial \psi}{\partial t} d t .
$$

The average stored energy $U$ is then

$$
U=\lim _{\sigma \rightarrow 0} \frac{\omega}{2 \pi} \int_{0}^{2 \pi / \omega} u(t) d t .
$$


Any term which is of double frequency, $2 \omega$, will not contribute to the average indicated in (43). Hence,

$$
U=\lim _{\sigma \rightarrow 0} \frac{1}{2}\left(\boldsymbol{\Phi}^{+}(p) \cdot((-i p \gamma(p)) / 2 \sigma) \boldsymbol{\Phi}(p) e^{2 \sigma t}\right)_{r} .
$$

But since, from (18), $\omega \gamma(\omega)$ is an analytic function of $\omega$ for $\omega$ in $\Delta$,

$$
p \gamma(p)=\omega \gamma(\omega)+\left(i \sigma \partial(\omega \gamma(\omega)) / \partial \omega+O\left(\sigma^{2}\right)\right)
$$

where $O\left(\sigma^{2}\right)$ denotes terms of the order of $\sigma^{2}$ as $\sigma \rightarrow 0$. When (45) is substituted into (44) one obtains (40).

From (40) it is clear that one can describe the inequality (38) as stating that the stored energy density for a given $\mathbf{E}(\omega)$ and $\mathbf{H}(\omega)$ is always greater than in free space.

In practice it is important to verify (38) as a check on the passivity of the medium. For a gyrotropic non-Tellegen medium with the $z$-axis taken in the direction of the DC magnetic field

$$
\epsilon=\epsilon_{0}\left(\begin{array}{ccc}
\epsilon_{1} & -i \epsilon_{2} & 0 \\
i \epsilon_{2} & \epsilon_{1} & 0 \\
0 & 0 & \epsilon_{3}
\end{array}\right), \quad \mu=\mu_{0}\left(\begin{array}{ccc}
\mu_{1} & -i \mu_{2} & 0 \\
i \mu_{2} & \mu_{1} & 0 \\
0 & 0 & \mu_{3}
\end{array}\right) .
$$

Equation (38) is then equivalent to

$$
\begin{gathered}
\frac{d}{d \omega}\left(\omega \epsilon_{1}\right) \geq 1, \quad \frac{d}{d \omega}\left(\omega \mu_{1}\right) \geq 1 \quad \frac{d}{d \omega}\left(\omega \epsilon_{3}\right) \geq 1, \quad \frac{d}{d \omega}\left(\omega \mu_{3}\right) \geq 1, \\
{\left[\frac{d}{d \omega}\left(\omega \epsilon_{1}\right)-1\right]^{2}-\left[\frac{d}{d \omega}\left(\omega \epsilon_{2}\right)\right]^{2} \geq 0, \quad\left[\frac{d}{d \omega}\left(\omega \mu_{1}\right)-1\right]^{2}-\left[\frac{d}{d \omega}\left(\omega \mu_{2}\right)\right]^{2} \geq 0}
\end{gathered}
$$

where at least one of these relations is a strict inequality. A typical example is the case of a ferrite. Here, [6], $\epsilon_{1}=\epsilon_{3}=1, \epsilon_{2}=0$ and

$$
\mu_{1}=1-\alpha \beta /\left(\omega^{2}-\beta^{2}\right), \quad \alpha \beta>0 \quad \mu_{2}=\omega \alpha /\left(\omega^{2}-\beta^{2}\right) .
$$

One readily verifies from (48) that

$$
d\left(\omega \mu_{1}\right) / d \omega=1+\alpha \beta\left(\left(\omega^{2}+\beta^{2}\right) /\left(\omega^{2}-\beta^{2}\right)^{2}\right)>1
$$

and

$$
\left[d\left(\omega \mu_{1}\right) / d \omega-1\right]^{2}-\left[d\left(\omega \mu_{2}\right) / d \omega\right]^{2}=(\alpha \beta)^{2} /\left(\omega^{2}-\beta^{2}\right)^{2}>0 .
$$

Hence (38) is valid. A second example is the case of a plasma. Here, [7], $\mu_{1}=\mu_{3}=1$, $\mu_{2}=0$ and

$$
\begin{aligned}
& \epsilon_{1}=1-\omega_{p}^{2} /\left(\omega^{2}-\omega_{c}^{2}\right), \\
& \epsilon_{2}=\left(\omega_{c} / \omega\right)\left(\omega_{p}^{2} /\left(\omega^{2}-\omega_{c}^{2}\right)\right), \\
& \epsilon_{3}=1-\omega_{p}^{2} / \omega^{2}
\end{aligned}
$$

where $\omega_{\nu}$ is the plasma frequency and $\omega_{c}$ is the cyclotron or gyro-frequency. One readily verifies from (49) that

$$
\begin{gathered}
d\left(\omega \epsilon_{1}\right) / d \omega=1+\omega_{p}^{2}\left(\left(\omega^{2}+\omega_{c}^{2}\right) /\left(\omega^{2}-\omega_{c}^{2}\right)^{2}\right)>1 \\
d\left(\omega \epsilon_{3}\right) / d \omega=1+\omega_{p}^{2} / \omega^{2}>1
\end{gathered}
$$


and

$$
\left[\left(d\left(\omega \epsilon_{1}\right) / d \omega\right)-1\right]^{2}-\left[\left(d\left(\omega \epsilon_{2}\right) / d \omega\right)\right]^{2}=\omega_{p}^{4} /\left(\omega^{2}-\omega_{c}^{2}\right)^{2}>0 .
$$

Hence (38) is again valid.

VI. Group velocity. A mode or eigenwave of a structure is a wave which can exist in the absence of any excitation. For a structure with translational symmetry vectors $a_{i}$ a typical mode can be characterized by phase-shift boundary conditions [13]

$$
\mathbf{E}\left(\mathbf{r}+\mathbf{a}_{i}\right)=\exp \left(i \mathbf{k} \cdot \mathbf{a}_{i}\right) \mathbf{E}(r) \text { and } \mathbf{H}\left(\mathbf{r}+\mathbf{a}_{\mathbf{i}}\right)=\exp \left(i \mathbf{k} \cdot \mathbf{a}_{\mathbf{i}}\right) \mathbf{H}(\mathbf{r})
$$

where the real "propagation" vector $\mathbf{k}$ is in the space $\mathcal{L}$, spanned by the $\mathbf{a}_{\mathbf{i}}$. If one defines $\mathrm{E}_{0}(\mathrm{r})$ and $\mathrm{H}_{0}(\mathrm{r})$ by

$$
\mathbf{E}(\mathbf{r})=\exp (i \mathbf{k} \cdot \mathbf{r}) \mathbf{E}_{0}(\mathbf{r}) \text { and } \mathbf{H}(\mathbf{r})=\exp (i \mathbf{k} \cdot \mathbf{r}) \mathbf{H}_{0}(\mathbf{r})
$$

then (50) is equivalent to the statement that $\mathrm{E}_{0}(\mathrm{r})$ and $\mathrm{H}_{0}(\mathrm{r})$ have the same periodicity as the structure, i.e.,

$$
\mathrm{E}_{0}\left(\mathbf{r}+\mathrm{a}_{i}\right)=\mathrm{E}_{0}(\mathbf{r}) ; \quad \mathrm{H}_{0}\left(\mathbf{r}+\mathbf{a}_{i}\right)=\mathrm{H}_{0}(\mathbf{r}) .
$$

When a structure is uniform in a direction a then $a_{1}=\alpha$ a will be a translational symmetry vector for $\alpha$ arbitrarily small. Hence, from (52), $\mathbf{E}_{0}(\mathbf{r})$ and $\mathrm{H}_{0}(\mathbf{r})$ will not vary in that direction. Thus for a structure uniform in all directions the modes in (51) are plane waves. In the general case the mode in (51), often described as a Bloch wave with propagation vector $\mathbf{k}$, appears to be a plane wave when sampled at points separated from each other by multiples of $a_{i}$.

The variation of $\mathbf{k}$ with frequency, i.e., the dispersiveness of the medium, is constrained by an equation which can be symbolized as

$$
f(\mathbf{k}, \omega)=0 .
$$

One refers to (53) as a dispersion equation (not to be confused with the dispersion relations (18)). From (53) one defines the group velocity, $\mathbf{v}_{\boldsymbol{g}}$, by

$$
\nabla_{0}=\partial \omega / \partial \mathbf{k} \text {. }
$$

(Some authors employ the notation $\nabla_{\mathbf{k}}$ or $\operatorname{grad}_{\mathbf{k}}$ instead of $\partial / \partial \mathbf{k}$.) Thus $\nabla_{0}$ is a vector in $\mathscr{L}$.

In order to identify $\mathbf{v}_{\boldsymbol{\theta}}$ as being an average rate of energy flow we use a basic identity, often referred to as a variational theorem [10]:

$$
\nabla \cdot(\overline{\mathbf{E}} \times \delta \mathrm{H}-\overline{\mathbf{H}} \times \delta \mathrm{E})=i \boldsymbol{\Phi}^{+} \delta(\omega \gamma) \mathbf{\Phi}
$$

where $\delta A$ denotes a variation in $A$. This result is well known for the case of a nonTellegen medium. The proof for a Tellegen medium is given in the Appendix. In general

$$
\delta A=(\partial A / \partial \omega) \delta \omega+(\partial A / \partial \mathbf{k}) \cdot \delta \mathbf{k} .
$$

Equation (55) can then be written as

$$
\nabla \cdot(\overline{\mathrm{E}} \times \delta \mathrm{H}-\overline{\mathrm{H}} \times \delta \mathrm{E})=4 i U \delta \omega
$$

where $U$ is defined in (40). When (51) is substituted into (57) one obtains, after a straightforward calculation,

$$
R+\delta \mathrm{k} \cdot \mathbf{r} \nabla \cdot \mathbf{P}+\delta \mathrm{k} \cdot \mathbf{P}=U \delta \omega
$$


where

$$
R=(1 / 4 i) \nabla \cdot\left(\overline{\mathrm{E}}_{0} \times \delta \mathrm{H}_{0}-\overline{\mathrm{H}}_{0} \times \delta \mathrm{E}_{0}\right) .
$$

In particular, when the medium is lossless, i.e., when $\gamma(\omega)$ is Hermitian, then, from (25), $\nabla \cdot \mathbf{P}=0$ and (58) reduces to

$$
\mathbf{R}+\delta \mathbf{k} \cdot \mathbf{P}=U \delta \omega .
$$

One now seeks to eliminate the term $R$ from (60). For simplicity we first discuss the plane wave modes of a uniform medium. In this case, $\mathbf{E}_{0}$ and $\mathbf{H}_{0}$ are independent of $\mathbf{r}$ and hence, from (59)

$$
R=0 \text {. }
$$

Equation (60) is then equivalent to

$$
\nabla_{0}=\mathbf{P}_{l} / U
$$

where $\mathbf{P}_{\boldsymbol{l}}$ denotes the component of $\mathbf{P}$ in the space $\mathscr{L}$ and $\mathbf{P}$ is defined in (22).

We now show from (62) that in a transparency interval of a passive dispersive medium the magnitude of $\boldsymbol{v}_{0}$ is less than $c$, i.e.,

$$
\left|\mathbf{v}_{0}\right|<c
$$

where $c$ is the velocity of light in free space.

For this purpose one notes the upper estimate of $\left|\mathbf{P}_{l}\right|$,

$$
\left|\mathbf{P}_{l}\right| \leq|\mathrm{P}| \leq \frac{1}{2}|\mathrm{E}||\mathbf{H}|
$$

and, from (38), the lower estimate of $U$,

$$
U>\frac{1}{4} \Phi^{+} \cdot \gamma_{0} \Phi=\frac{1}{4}\left(\epsilon_{0}|\mathbf{E}|^{2}+\mu_{0}|\mathbf{H}|^{2}\right) \text {. }
$$

But if $a$ and $b$ are any positive real numbers then

$$
(a b)^{1 / 2} \leq \frac{1}{2}(a+b)
$$

i.e., the geometric mean is less than or equal to the arithmetic mean. In particular, if

$$
a=\frac{1}{2} \epsilon_{0}|\mathbf{E}|^{2} \text { and } b=\frac{1}{2} \mu_{0}|\mathbf{H}|^{2}
$$

then (66) becomes

$$
\frac{1}{2}\left(\mu_{0} \epsilon_{0}\right)^{1 / 2}|\mathrm{E}||\mathbf{H}| \leq \frac{1}{4}\left(\epsilon_{0}|\mathbf{E}|^{2}+\mu_{0}|\mathbf{H}|^{2}\right)
$$

or, in view of (64), (65) and (6)

$$
\left|\mathbf{P}_{l}\right| / c<U
$$

In view of (62), this is clearly equivalent to (63).

For modes of more general structures one first defines a unit cell as follows: Two points in space are said to be equivalent if their difference is a translational symmetry vector. A (closed) unit cell is then a smallest (closed) connected set of points with the property that every point in the structure has an equivalent point in the unit cell. More specifically, let $a_{1}, \cdots, a_{m}$ be $m$ linearly independent translational symmetry vectors where $m$ is 1,2 or 3 . Let $\&$ be the $m$-dimensional space containing the $a_{i}$. The vector $\mathbf{r}$ can then be represented as 


$$
\mathbf{r}=\left(\mathbf{r}_{\imath}, \mathbf{r}_{\imath}\right)
$$

where $r_{l}$ is the component of $\mathbf{r}$ in $\mathscr{L}$ and $\mathbf{r}_{t}$ is the component out of $\mathscr{L}$. One refers to $\mathbf{r}_{l}$ as the longitudinal or lattice component and to $r_{t}$ as the transverse or cross-sectional component of $\mathbf{r}$. When $\mathbf{r}_{l}$ is kept constant, the range of values of $\mathbf{r}_{\mathfrak{l}}$ define a cross-section of the structure.

A general representation for $\mathbf{r}_{l}$ is

$$
\mathbf{r}_{l}=\alpha_{1} \mathbf{a}_{1}+\cdots+\alpha_{m} a_{m}
$$

where each $\alpha_{i}$ ranges over all real numbers from $-\infty$ to $+\infty$. One obtains the points of a closed unit cell by restricting the $\alpha_{i}$ by, say,

$$
\left|\alpha_{i}\right| \leq 1 / 2 \text {. }
$$

A unit cell of the structure is then characterized by $\mathbf{r}_{l}$ ranging over a unit cell of $\mathfrak{L}$ and, for each such $r_{l}, r_{t}$ ranging over the entire cross section.

As an example, consider a structure modulated periodically in directions $a_{1}$ and $a_{2}$ perpendicular to the $z$-axis. In this case $\mathbf{r}_{t}$ is simply the $z$-component of $\mathbf{r}$ and a typical cross-section is a line parallel to the $z$-axis between the boundaries of the structure. A unit cell in $\mathcal{L}$ is then, for example, a parallelepiped with sides $\mathbf{a}_{1}$ and $\mathbf{a}_{2}$. In particular, if $a_{1}$ and $a_{2}$ can be taken arbitrarily small in magnitude, i.e., if the structure is plane stratified, then a unit cell in $\mathscr{L}$ is a single point and a unit cell of the structure is any cross-section (cf. [14]).

When (59) is integrated over a unit cell one obtains from the divergence theorem

$$
\int R d v=\int_{b} \mathbf{n} \cdot\left(\overline{\mathbf{E}}_{0} \times \delta \mathbf{H}_{0}-\overline{\mathbf{H}}_{0} \times \delta \overline{\mathbf{H}}_{0}\right) d s / 4 i
$$

where $b$ is the boundary of the unit cell and $\mathbf{n}$ is the unit exterior normal. Instead of (61) one then often has

$$
\int R d v=0
$$

where the integration in (73) is over a unit cell. The reason for (73) can be seen as follows: At equivalent points of $b, \mathbf{n}$, being an outward normal, is reversed in direction. Furthermore, from (52)

$$
\delta \mathrm{E}_{0}\left(\mathbf{r}+\mathbf{a}_{i}\right)=\delta \mathrm{E}_{0}(\mathbf{r}) ; \quad \delta \mathrm{H}_{0}\left(\mathbf{r}+\mathbf{a}_{i}\right)=\delta \mathrm{H}_{0}(\mathbf{r}) .
$$

Hence, the contribution from the integral in (72) from equivalent portions of $b$ will cancel. This argument fails for portions of $b$ which have no equivalent points in $b$. But then they must be on the boundary of the structure and the boundary conditions will often be such that the integrand in (72) vanishes. Such boundary conditions are, for example, that portions of $b$ should consist of electric walls, where

$$
\mathrm{n} \times \mathrm{E}=0
$$

or magnetic walls, where

$$
\mathrm{n} \times \mathrm{H}=0
$$

or infinitely distant walls, where

$$
\mathrm{E} \rightarrow 0 \text { and } \mathrm{H} \rightarrow 0
$$


"sufficiently" rapidly. When (60) is then integrated over a unit cell one obtains, in view of (73),

$$
\delta \mathbf{k} \cdot \int \mathbf{P} d v=\int U d v \delta \omega
$$

which is equivalent to

$$
\mathbf{v}_{o}=\int \mathbf{P}_{l} d v / \int U d v
$$

But clearly

$$
\left|\int \mathbf{P}_{\iota} d v\right| \leq \int\left|\mathbf{P}_{l}\right| d v
$$

and from (6s)

$$
\int\left|\mathbf{P}_{l}\right| d v<c \int U d v
$$

Hence, from (79), (80) and (81) the inequality (63) is again valid.

When the unit cell has a finite volume, $V$, then one can divide both the numerator and denominator in (79) by this volume to obtain

$$
\mathbf{v}_{0}=\text { average } \mathbf{P}_{l} / \text { average } U
$$

where

$$
\text { average } A=\int A d v / V .
$$

Actually, (82) includes (62) as a special case since, as discussed above, a unit cell for a structure uniform in all directions is simply a point in space and

$$
\text { average } A \rightarrow A \text { as } V \rightarrow 0
$$

at points where $A$ is continuous.

A boundary condition more general than (75) or (76) is [1]

$$
\mathrm{n} \times \mathrm{E}_{\imath}=\mathrm{ZH}_{\mathrm{t}}
$$

or, equivalently,

$$
\mathrm{E}_{\mathrm{t}}=Z \mathrm{H}_{\mathbf{t}} \times \mathrm{n}
$$

where $\mathbf{A}_{\boldsymbol{t}}$ denotes the component of $\mathbf{A}$ tangential to the surface and $Z$ is a surface impedance dyad. (In any particular coordinate system $Z$ is represented by a matrix.)

One can analyze $Z$ in a manner analagous to the discussion of $\gamma$ in the preceding sections. Thus a surface impedance is passive at frequency $\omega$ if

$$
Z_{h} \geq 0
$$

and is lossless if

$$
Z_{h}=0 .
$$

This follows since $\mathbf{P} \cdot \mathbf{n}$ is the average power dissipated in the surface and $2 \mathbf{P} \cdot \mathbf{n}=$ 
$\left.(\mathbf{E} \times \overline{\mathbf{H}})_{r} \cdot \mathbf{n}=\left(\overline{\mathbf{H}}_{t} \cdot \mathbf{n} \times \mathrm{E}_{t}\right)_{v}=\overline{\mathbf{H}}_{t} \cdot Z \mathbf{H}\right)_{r}^{\prime}$ i.e.,

$$
2 \mathrm{P} \cdot \mathbf{n}=\mathrm{H}_{t} \cdot Z_{h} \mathrm{H}_{\mathbf{t}} .
$$

Equations (86) and (87) are an immediate corollary of (88). One can rewrite the lossless case (87) as

$$
Z=-i X \text { with } X=X^{+} .
$$

The average stored energy density, $U_{b}$, in the surface is

$$
U_{b}=\frac{1}{4} \overline{\mathbf{H}}_{t} \cdot \frac{\partial X}{\partial \omega} \mathbf{H}_{\imath} .
$$

This is readily seen from the complex frequency approach employed in Sec. V.

From (51) and (85) one obtains, after a straightforward calculation,

$$
\mathbf{n} \cdot\left(\overline{\mathbf{E}}_{0} \times \delta \mathrm{H}_{0}-\overline{\mathbf{H}}_{0} \times \delta \mathrm{E}_{0}\right)=\overline{\mathbf{H}}_{0 t} \cdot\left(Z+Z^{+}\right) \mathbf{H}_{0 t}+\overline{\mathbf{H}}_{0 t} \cdot \delta Z \mathrm{H}_{0 \iota} .
$$

In particular, when the surface impedance is lossless, i.e., when $Z+Z^{+}=0$, and for the moment neglecting any dependence of $Z$ on $\mathbf{k}$, one obtains from (90) and (91)

$$
\mathbf{n} \cdot\left(\overline{\mathbf{E}}_{0} \times \delta \mathrm{H}_{0}-\overline{\mathbf{H}}_{0} \times \delta \mathbf{E}_{0}\right)=-4 i U_{b} \delta \omega .
$$

In view of $(92),(7 \gamma)$ is modified to

$$
\delta \mathbf{k} \cdot \int \mathbf{P} d v=\int U d v+\int_{b} U_{b} d s
$$

which is equivalent to

$$
\mathbf{v}_{o}=\int \mathbf{P}_{l} d v /\left(\int U d v+\int_{b} U_{b} d s\right) .
$$

Since both $U$ and $U_{b}$ are nonnegative, one obtains an upper bound for $\left|\mathbf{v}_{a}\right|$ by neglecting the boundary integral in the denominator of (94). Thus (63) is also valid in this case.

Finally, when one allows a dependence upon $\mathbf{k}$ in $\gamma$ (as occurs in a hot plasma [9], [10]) and in $Z$ (as in [14]) then (55) is still valid, (60) is modified to

$$
R+\delta \mathbf{k} \cdot\left(\mathbf{P}+\mathbf{P}_{\gamma}\right)=U \delta \omega
$$

where

$$
\mathbf{P}_{\gamma}=-\frac{1}{4} \boldsymbol{\Phi}^{+} \cdot \omega(\partial \gamma / \partial \mathbf{k}) \mathbf{\Phi}
$$

and (92) is modified to

$$
\mathbf{n} \cdot\left(\overline{\mathbf{E}}_{0} \times \delta \mathbf{H}_{0}-\overline{\mathbf{H}}_{0} \times \delta \mathbf{E}_{0}\right)=-4 i U_{b} \delta \omega+4 i \mathbf{P}_{b} \cdot \delta \mathbf{k}
$$

where

$$
\mathbf{P}_{b}=-\frac{1}{4} \overline{\mathbf{H}}_{0 \iota} \cdot(\partial X / \partial \mathbf{k}) \mathbf{H}_{0 t} .
$$

The formula for $\mathbf{v}_{0}$ then turns out to be

$$
\mathbf{\nabla}_{o}=\left(\int\left(\mathbf{P}+\mathbf{P}_{\gamma}\right)_{l} d v+\int_{b}\left(\mathbf{P}_{b}\right)_{l} d S\right) / \int U d v+\int_{b} U_{b} d S .
$$

In this case the proof of (63) requires that dispersion relations with respect to both $\omega$ and $\mathbf{k}[20]$ be employed. 


\section{Appendix}

\section{PROOF OF THE VARIATION THEOREM FOR A TELLEGEN MEDIUM}

Let

$$
\sigma=i\left[\begin{array}{cc}
0 & I \\
-I & 0
\end{array}\right]
$$

Then

$$
\sigma=\sigma^{+}
$$

and

$$
\sigma=\sigma^{-1}
$$

The Maxwell equations are

$$
\nabla \times \boldsymbol{\Phi}=\sigma \omega \gamma \boldsymbol{\Phi}
$$

where

$$
\boldsymbol{\Phi}=\left[\begin{array}{l}
\mathbf{E} \\
\mathbf{H}
\end{array}\right] \text { and } \nabla \times \mathbf{\Phi}=\left[\begin{array}{c}
\nabla \times \mathbf{E} \\
\nabla \times \mathbf{H}
\end{array}\right] .
$$

In view of $(A-1)$ and $(A-3)$ one can rewrite $(A-4)$ as

$$
\nabla \times \sigma \boldsymbol{\Phi}=\omega \gamma \boldsymbol{\Phi} .
$$

Taking the variation of (A-6) one obtains

$$
\nabla \times \sigma \delta \Phi=\delta(\omega \gamma) \Phi+\omega \gamma \delta \Phi
$$

and hence

$$
\boldsymbol{\Phi}^{+} \cdot(\nabla \times \sigma \delta \Phi)=\Phi^{+} \cdot \delta(\omega \gamma) \Phi+\boldsymbol{\Phi}^{+} \cdot \omega \gamma \delta \boldsymbol{\Phi} .
$$

Taking the adjoint of the Maxwell equations (A-4) one obtains

$$
\nabla \times \boldsymbol{\Phi}^{+}=\boldsymbol{\Phi}^{+} \omega \gamma^{+} \sigma^{+}
$$

and hence, in view of $(\mathrm{A}-2)$ and $(\mathrm{A}-3)$,

$$
\nabla \times \boldsymbol{\Phi}^{+} \cdot \sigma \delta \Phi=\Phi^{+} \omega \gamma^{+} \cdot \delta \Phi .
$$

But

$$
\nabla \cdot\left(\boldsymbol{\Phi}^{+} \times \sigma \delta \boldsymbol{\Phi}\right)=\nabla \times \boldsymbol{\Phi}^{+} \cdot \sigma \delta \boldsymbol{\Phi}-\boldsymbol{\Phi}^{+} \cdot \nabla \times \sigma \delta \boldsymbol{\Phi}
$$

is a vector identity. Hence the result of subtracting (A-7) from (A-8) is

$$
\nabla \cdot\left(\boldsymbol{\Phi}^{+} \times \sigma \delta \boldsymbol{\Phi}\right)=\boldsymbol{\Phi}^{+} \cdot \omega\left(\gamma^{+}-\gamma\right) \delta \boldsymbol{\Phi}-\boldsymbol{\Phi}^{+} \cdot \delta(\omega \gamma) \boldsymbol{\Phi} .
$$

But by definitions (A-1) and (A-5)

$$
\nabla \cdot\left(\boldsymbol{\Phi}^{+} \times \sigma \delta \boldsymbol{\Phi}\right)=i \nabla \cdot(\overline{\mathrm{E}}, \overline{\mathrm{H}}) \times\left|\begin{array}{r}
\delta \mathrm{H} \\
-\delta \mathrm{E}
\end{array}\right|=i \nabla \cdot(\overline{\mathrm{E}} \times \delta \mathrm{H}-\overline{\mathrm{H}} \times \delta \mathrm{E}) .
$$


Hence if $\gamma=\gamma^{+}$one can rewrite (A-10) with the aid of (A-11) as

$$
\nabla \cdot(\overline{\mathrm{E}} \times \delta \mathrm{H}-\overline{\mathrm{H}} \times \delta \mathrm{E})=i \boldsymbol{\Phi}^{+} \cdot \delta(\omega \gamma) \boldsymbol{\Phi} .
$$

Q.E.D.

\section{REFERENCES}

[1] L. D. Landau and E. M. Lifshitz, Electrodynamics of continuous media, Pergamon, New York, 1960, pp. 247-267

[2] L. Page and N. I. Adams, Electrodynamics, D. Van Nostrand, New York, 1940, p. 405

[3] B. D. H. Tellegen, The gyrator, a new electric network element, Philips Res. Rep., 3, 81-101 (1948)

[4] LL. G. Chambers, Propagation in a gyrational medium, Quart J. Mech. Appl. Math. 9, 360-370 (1956)

[5] B. S. Gourary, Dispersion relations for tensor media and their application to ferrites, J. Appl. Phys. 28, 283-288 (1957)

[6] D. Polder, On the theory of ferromagnetic resonance, Phil. Mag. 40, 99-115 (1949)

[7] E. Astrom, On waves in an ionized gas, Arkiv Fysik 2, 443-457 (1951)

[8] C. O. Hines, Wave packets, the Poynting vector and energy flow, J. Geophysics Res. 56, 63, 107, 207, $535(1951)$

[9] T. H. Stix, The theory of plasma waves, McGraw-Hill, New York, 1962, pp. 45-52

[10] W. P. Allis, S. J. Buchsbaum and A. Bers, Waves in anistotropic plasmas, M.I.T. Press, Cambridge, Mass., 1963, pp. 95-131

[11] F. E. Borgnis and C. H. Papas, Handbuch der Physik, Springer, Berlin, 1958, p. 289 and p. 312

[12] D. A. Watkins, Topics in electromagnetic theory, Wiley, New York, 1958, pp. 12-14

[13] E. H. Wagner, Uber Gruppengeschwindigkeit, Energiestromdichte und Energiedichte in der Rontgenbezw Lichtoptik der Kristalle, Zeit. fur Physik 154, 352-360 (1959)

[14] H. L. Bertoni and A. Hessel, Group velocity and power flow relations for surface waves in planestratified anisotropic media, IEEE Trans. on Antennas and Propagation, AP-14, 344-352 (1966)

[15] C. G. Montgomery, R. H. Dicke and E. M. Purcell, Principles of microwave circuits, McGraw-Hill, New York, 1948, pp. 151-152

[16] J. S. Toll, Causality and the dispersion relation: logical foundations, Phys. Rev. 104, 1760-1770 (1956)

[17] J. A. Stratton, Electromagnetic theory, McGraw-Hill, New York, 1941, p. 137

[18] L. Brillouin, Wave propagation and group velocity, Academic Press, New York, 1960, pp. 88-93

[19] A. Tonning, Energy density in continuous electromagnetic media, IRE Trans. on Antennas and Propagation, AP-8, 428-434 (1960)

[20] M. Leontovitch, Generalization of the Kramers-Kronig formulas to media with spatial dispersion, Soviet Physics-JETP 13, 634-637 (1961) 